\title{
Secondary osteosarcoma in patients previously treated for childhood cancer: Three case reports
}

\author{
AKIYOSHI SHIMATANI ${ }^{1}$, MASANARI AONO ${ }^{2}$, MANABU HOSHI $^{1}$, NAOTO OEBISU $^{1}$, \\ TADASHI IWAI ${ }^{1}$, NAOKI TAKADA ${ }^{1}$, JUNICHI HARA $^{3}$, CHIKA NITANI $^{3}$ and HIROAKI NAKAMURA ${ }^{1}$ \\ ${ }^{1}$ Department of Orthopedic Surgery, Osaka City University Graduate School of Medicine, Osaka, Osaka 545-8585; \\ Departments of ${ }^{2}$ Orthopedic Surgery and ${ }^{3}$ Pediatric Hematology and Oncology, \\ Osaka City General Hospital, Osaka, Osaka 534-0021, Japan
}

Received September 6, 2018; Accepted October 24, 2018

DOI: $10.3892 /$ mco.2018.1752

\begin{abstract}
The prognosis of childhood cancers has improved markedly, and the proportion of long-term survivors has increased in recent years. However, with the increase in the number of long-term survivors, the development of latent treatment-related adverse effects, such as secondary malignancies, has generated new problems. Secondary cancer is defined as a histologically distinct malignancy that develops at least 2 months after the completion of treatment for primary cancer. Genetic factors and acquired conditions associated with treatment modalities are possible causes of secondary malignancy development. Genetic factors include the presence of Li-Fraumeni syndrome (LFS) and retinoblastoma. In terms of acquired factors, radiation and chemotherapy have been reported to be the most strongly associated with secondary malignancy development. The use of alkylating agents and topoisomerase II inhibitors for the treatment of childhood cancer increases the subsequent risk of secondary tumors. We herein investigated three cases of secondary osteosarcoma several years after treatment for primary cancer. In the three patients, the familial history did not appear to fit the clinical diagnostic criteria of LFS or retinoblastoma. The patients had not received previous radiation therapy to the anatomical site of the secondary cancer. However, high dosages of alkylating agents and topoisomerase II inhibitors had been administered for the treatment of primary cancer. The exact link between chemotherapy and secondary cancer remains elusive, but the possibility of an association should be considered. Following
\end{abstract}

Correspondence to: Dr Akiyoshi Shimatani, Department of Orthopedic Surgery, Osaka City University Graduate School of Medicine, 1-4-3 Asahi-Machi, Abeno-ku, Osaka, Osaka 545-8585, Japan

E-mail: bpcip920@tcct.zaq.ne.jp

Abbreviations: MR, magnetic resonance; CT, computed tomography

Key words: secondary cancer, childhood cancer, chemotherapy, latent adverse effect, osteosarcoma the development of multidisciplinary therapies, long-term follow-up and monitoring of latent adverse effects may be necessary for childhood cancer survivors.

\section{Introduction}

The prognosis of childhood cancer has improved significantly, and the number of long-term survivors has increased in recent years, mainly due to improvements in multidisciplinary therapy, including neoadjuvant chemotherapy, radiation therapy and surgical techniques. With the increase in the number of long-term survivors, the development of latent treatment-related adverse effects, such as secondary cancer, has generated new problems.

Secondary cancers are defined as histologically distinct malignancies that develop at least 2 months after the completion of treatment for primary cancer. Secondary cancers occur due to genetic factors, as well as previous chemotherapy and/or radiation therapy.

We herein present three cases of secondary osteosarcoma that developed after treatment for childhood cancer in our hospital (Tables I and II) and report on their characteristic clinical features, diagnosis, treatment and outcomes.

The patients and their parents provided their consent for the publication of patient data.

\section{Case reports}

Case 1. An 11-year-old male patient experienced pain in the left thigh for $>2$ months. The family history provided no indication of a genetic predisposition to bone tumors. When the patient was 4 years old, he developed medulloblastoma in the cerebellar vermis and was treated by tumor resection and, postoperatively, whole-brain irradiation (18 Gy), localized irradiation (32 Gy) and high-dose intensive chemotherapy. The patient was administered four cycles of chemotherapy with vincristine, cyclophosphamide, cisplatin and etoposide, and one cycle with thiotepa and melphalan. The patient also received six intrathecal injections of dexamethasone and methotrexate. Furthermore, etoposide was administered for 2 years due to intrathecal metastasis. The patient remained disease-free for 6 years and 3 months after the last cycle of chemotherapy. 
Six years after treatment for medulloblastoma, the patient experienced gradually increasing pain in the left thigh. At initial hospital presentation, muscle pain was suspected, as a plain radiograph revealed no abnormalities. However, the pain persisted. The patient visited another hospital 2 months after symptom onset, and a bone tumor of the left femur was identified on X-ray imaging. At that point, the patient was referred to Osaka City General Hospital (Osaka, Japan) on December 2008. X-ray imaging revealed a lytic lesion with spiculated periosteal reaction in the left proximal femoral shaft (Fig. 1A). Coronal T1-weighted and T2-weighted magnetic resonance (MR) images revealed a heterogeneously low-intense (T1) and heterogeneously hyperintense (T2) mass extending from the proximal femoral bone marrow to the adjacent soft tissues (Fig. 1B). Pathological diagnosis of the biopsy specimen from the extraosseous lesions revealed chondroblastic osteosarcoma, with spindle cells in the cartilage lacunae and a cartilage-like matrix (Fig. 1C). One cycle of chemotherapy with adriamycin $\left(60 \mathrm{mg} / \mathrm{m}^{2}\right)$ and cisplatin $\left(100 \mathrm{mg} / \mathrm{m}^{2}\right)$, and another with ifosfamide $\left(10 \mathrm{~g} / \mathrm{m}^{2}\right)$ were administered. However, the tumor progressed due to treatment resistance. Consequently, a wide resection of the bone tumor and reconstruction with artificial implants were performed. The definitive diagnosis was chondroblastic osteosarcoma, and the secondary cancer was suspected to be associated with chemotherapy, as the histopathological pattern was different from that of the primary cancer (Fig. 1D). Postoperatively, two cycles of chemotherapy with methotrexate $\left(132 \mathrm{~g} / \mathrm{m}^{2}\right)$, pirarubicin $\left(40 \mathrm{mg} / \mathrm{m}^{2}\right)$, carboplatin $\left(400 \mathrm{mg} / \mathrm{m}^{2}\right)$, irinotecan $\left(100 \mathrm{mg} / \mathrm{m}^{2}\right)$ and ifosfamide $\left(10 \mathrm{~g} / \mathrm{m}^{2}\right)$ were administered. At the last follow-up, on February 2015, the patient had remained disease-free for 6 years.

Case 2. A 14-year-old male patient experienced pain in the right knee for $>3$ months. The family history provided no indication of a genetic predisposition to bone tumors. When the patient was aged 9 years, he developed a germ cell tumor in the anterior mediastinum (Fig. 2A). Preoperatively, the patient was treated with high-dose intensive chemotherapy including three cycles of bleomycin, etoposide and cisplatin, one cycle of paclitaxel, ifosfamide and cisplatin, and one cycle of etoposide and carboplatin (Table I). Postoperatively, two cycles of chemotherapy with paclitaxel and ifosfamide, and two cycles with etoposide and carboplatin were administered. The patient remained disease-free for 4 years and 6 months after the last cycle of chemotherapy.

Five years after receiving treatment for the germ cell tumor, the patient experienced gradually increasing pain in the right knee. The presence of a bone tumor was confirmed at a local hospital, followed by immediate referral to Osaka City General Hospital (Osaka, Japan) in February 2013. $\mathrm{X}$-ray imaging revealed a lytic lesion in the distal right femur (Fig. 2B). MR scanning was not performed due to the introduction of a stainless-steel wire in the sternum during the previous surgery. Computed tomography (CT) revealed the presence of an osteolytic lesion in the distal femoral metaphysis. Pathological diagnosis of the biopsy specimen from the lesion confirmed malignancy, suspected to be a sarcomatoid carcinoma. Preoperatively, the patient received two cycles of chemotherapy. He then underwent wide resection of the tumor and reconstruction with artificial knee joint replacement. The definitive diagnosis was osteoblastic osteosarcoma (Fig. 2C), and secondary cancer associated with chemotherapy was suspected, as the histopathological pattern was different from that of the primary cancer (Fig. 2D). Postoperatively, four cycles of chemotherapy were administered with $240 \mathrm{mg} / \mathrm{m}^{2}$ cyclophosphamide, $36 \mathrm{~g} / \mathrm{m}^{2}$ ifosfamide, $450 \mathrm{mg} / \mathrm{m}^{2}$ pirarubicin and $132 \mathrm{~g} / \mathrm{m}^{2}$ methotrexate pre- and postoperatively, according to CCG-7921 and POG-9351 (1). At the last follow-up, on March 2015, the patient had remained disease-free for 2 years.

Case 3. A 9-year-old male patient experienced pain in the right knee for $>1$ month. The family history provided no indication of a genetic predisposition to bone tumors. At the age of 1 year, the patient developed anaplastic astrocytoma in the medulla oblongata and was treated with localized brain irradiation (40 Gy) and high-dose intensive chemotherapy. Four cycles of chemotherapy with vincristine, cyclophosphamide, cisplatin, thiotepa and melphalan, and 14 cycles of etoposide were administered (Table I). The patient remained disease-free for 6 years after the last cycle of chemotherapy.

Eight years after treatment for the primary cancer, the patient experienced gradually increasing pain in the right knee. At first, sciatica was suspected at a local hospital. Sagittal T1-weighted and sagittal T2-weighted MR images revealed a heterogeneously hyperintense (T1) and isointense (T2) mass in the intradural region at the L3 level (Fig. 3A). Astrocytoma recurrence was suspected, and the patient was referred to Osaka City General Hospital (Osaka, Japan) in March 2010. X-ray imaging revealed an ossifying lesion with periosteal reaction in the proximal right tibia. Coronal T1-weighted and T2-weighted MR images revealed a heterogeneously hypointense (T1) and heterogeneously hyperintense (T2) mass extending from the proximal tibial metadiaphysis to the adjacent soft tissues (Fig. 3B). The patient underwent intradural tumor resection and anaplastic astrocy toma was pathologically diagnosed (Fig. 3C); however, the pathological diagnosis of the biopsy specimen from the right proximal tibial lesion was osteoblastic osteosarcoma (Fig. 3D). High-dose intensive chemotherapy, including ifosfamide $\left(6 \mathrm{~g} / \mathrm{m}^{2}\right)$, pirarubicin $\left(40 \mathrm{mg} / \mathrm{m}^{2}\right)$ and cisplatin (100 $\left.\mathrm{mg} / \mathrm{m}^{2}\right)$, was administered. However, due to rapid tumor growth, thigh amputation was performed 2 months after admission. Despite subsequent multiple regimens of aggressive chemotherapy including pirarubicin and cisplatin, ifosfamide and etposide, and etposide and temozolomide, the tumor progressed quickly. The patient eventually succumbed to disseminated metastatic disease, including the worsening of lung metastasis in September 2010.

\section{Discussion}

We herein report three cases of secondary osteosarcoma that developed after treatment for a childhood cancer. Of all the primary malignant bone tumors, osteosarcoma is the most common, accounting for $19.2 \%$ of all primary malignant bone tumors. However, its absolute incidence is low, at 5.6 cases per million population annually (2-4).

While osteosarcoma may occur as a secondary malignancy during childhood, its incidence is very low. In the large-scale 
Table I. Primary cancer.

\begin{tabular}{|c|c|c|c|c|c|}
\hline Case & Age, years & Histology & Site & Chemotherapy, cumulative doses & Radiation \\
\hline 1 & 4 & Medulloblastoma & Cerebellar vermis & $\begin{array}{l}\text { VCR, } 0.6 \mathrm{mg} / \mathrm{m}^{2}, \mathrm{CPM}, 12,000 \mathrm{mg} / \mathrm{m}^{2} \\
\text { CDDP, } 360 \mathrm{mg} / \mathrm{m}^{2}, \mathrm{VP} 16,14,000 \mathrm{mg} / \mathrm{m}^{2} \\
\text { TEPA, } 800 \mathrm{mg} / \mathrm{m}^{2}, \mathrm{LPAM}, 280 \mathrm{mg} / \mathrm{m}^{2}, \\
\text { MTX (intrathecal injection), } 72 \mathrm{mg} / \text { body }\end{array}$ & $\begin{array}{l}\text { Yes } \\
32 \text { Gy (brain) } \\
18 \text { Gy (spine) }\end{array}$ \\
\hline 2 & 9 & Germ cell tumor & Anterior mediastinum & $\begin{array}{l}\text { IFM, } 18 \mathrm{~g} / \mathrm{m}^{2}, \text { CBDCA }, 4,500 \mathrm{mg} / \mathrm{m}^{2} \\
\text { CDDP, } 400 \mathrm{mg} / \mathrm{m}^{2}, \text { VP-16, } 5,100 \mathrm{mg} / \mathrm{m}^{2} \\
\text { BLM, } 45 \mathrm{mg} / \mathrm{m}^{2}, \text { PTX, } 650 \mathrm{mg} / \mathrm{m}^{2}\end{array}$ & None \\
\hline 3 & 1 & $\begin{array}{l}\text { Anaplastic } \\
\text { astrocytoma }\end{array}$ & Medulla oblongata & $\begin{array}{l}\text { VCR, CPM, CDDP, VP-16, TEPA, L-PAM } \\
\text { (Cumulative doses are not applicable) }\end{array}$ & $\begin{array}{l}\text { Yes } \\
40 \text { Gy (brain) }\end{array}$ \\
\hline
\end{tabular}

VCR, vincristine; CPM, cyclophosphamide; CDDP, cisplatin; VP16, etoposide; TEPA, thiotepa; LPAM, melphalan; MTX, methotrexate; IFM, ifosfamide; CBDCA, carboplatin; BLM, bleomycin; PTX, paclitaxel.

Table II. Secondary cancer.

\begin{tabular}{lllll}
\hline Case & Interval & \multicolumn{1}{c}{ Histology } & \multicolumn{1}{c}{ Site } & Prognosis follow-up \\
\hline 1 & 6 years & Chondroblastic osteosarcoma & Proximal left femur & CDF 6 years \\
2 & 5 years & Osteoblastic osteosarcoma & Distal right femur & CDF 2 years \\
3 & 8 years & Osteoblastic osteosarcoma & Proximal right tibia & DOD 6 months \\
\hline
\end{tabular}

CDF, continuous disease free; DOD, dead of disease.
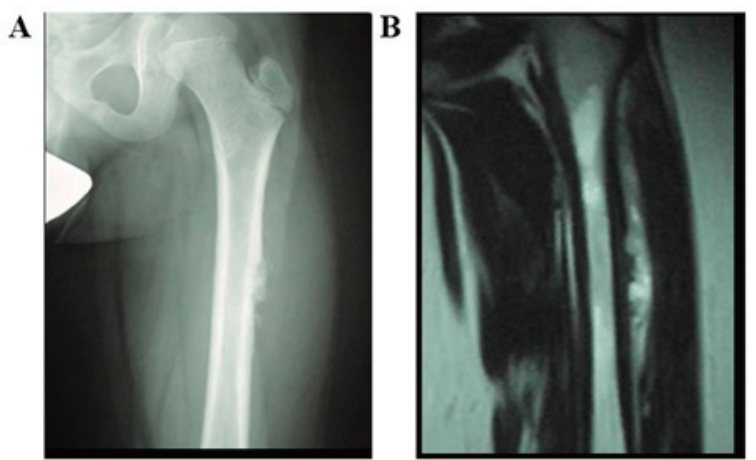

C
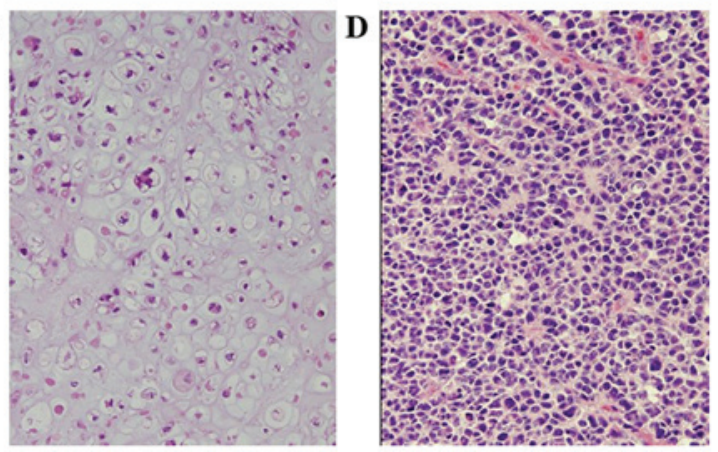

Figure 1. Case 1. (A) Radiograph of the left proximal femur demonstrating an aggressive lytic bone lesion with spiculated periosteal reaction in the proximal femoral metadiaphysis. (B) Coronal T2-weighted magnetic resonance imaging showing heterogeneous expansive mass, with high signal intensity. (C) Pathological examination confirmed chondroblastic osteosarcoma (hematoxylin and eosin staining; magnification, x200). (D) Primary cancer: Medulloblastoma of the cerebellar vermis (hematoxylin and eosin staining; magnification, $\mathrm{x} 200$ ).
Childhood Cancer survey, osteosarcoma as a secondary malignancy was reported in 31 of 14,372 survivors of childhood cancer (5). The incidence of osteosarcoma as a secondary malignancy following childhood cancer is expected to rise accordingly, due to the increase in the number of childhood cancer survivors. While primary osteosarcoma treatment is based on a clearly defined protocol including chemotherapy and surgery, the treatment protocol for osteosarcoma as a secondary neoplasm is not well-established. The 5-year survival rate associated with conventional osteosarcoma is reportedly $60-70 \%(6,7)$. The prognosis of patients with secondary bone sarcomas is generally poor. However, Bielack et al (8) reported that the prognosis of secondary osteosarcoma may increase to match that of the otherwise comparable primary osteosarcoma. In that study, chemotherapy was administered according to the contemporary Cooperative Osteosarcoma Study Group protocols for secondary osteosarcoma (9), including surgery and multiagent chemotherapy. Furthermore, Yonemoto et al (10) reported that 7 of 9 patients $(77.8 \%)$ with osteosarcoma as a secondary cancer survived without disease (mean follow-up period, 10.9 years); therefore, the prognosis of osteosarcoma occurring as a secondary malignancy following treatment for childhood cancer may be more favorable compared with that of conventional osteosarcoma.

Secondary cancer is defined as a histologically distinct malignancy, developing at least 2 months after the completion of treatment for the primary cancer (11). The cumulative incidence of secondary cancer is $15 \%$ at 20 years after the diagnosis of the primary cancer, and the incidence risk remains increased 

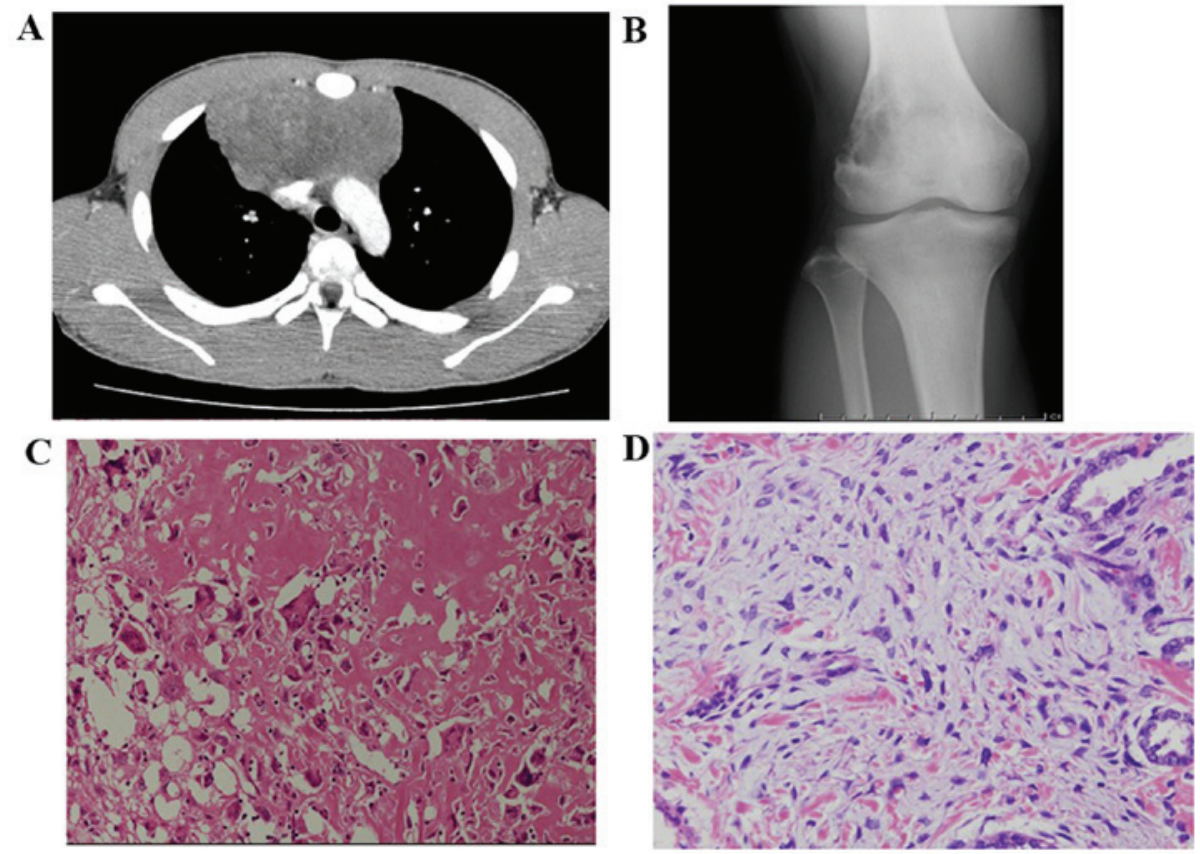

Figure 2. Case 2. (A) Computed tomography revealed a tumor in the anterior mediastinum. (B) X-ray of the right distal femur showing an aggressive lytic bony lesion in the distal femoral metaphysis. (C) Pathological examination confirmed osteoblastic osteosarcoma (hematoxylin and eosin staining; magnification, x200). (D) Primary cancer: Germ cell tumor in the anterior mediastinum (hematoxylin and eosin staining; magnification, x200).
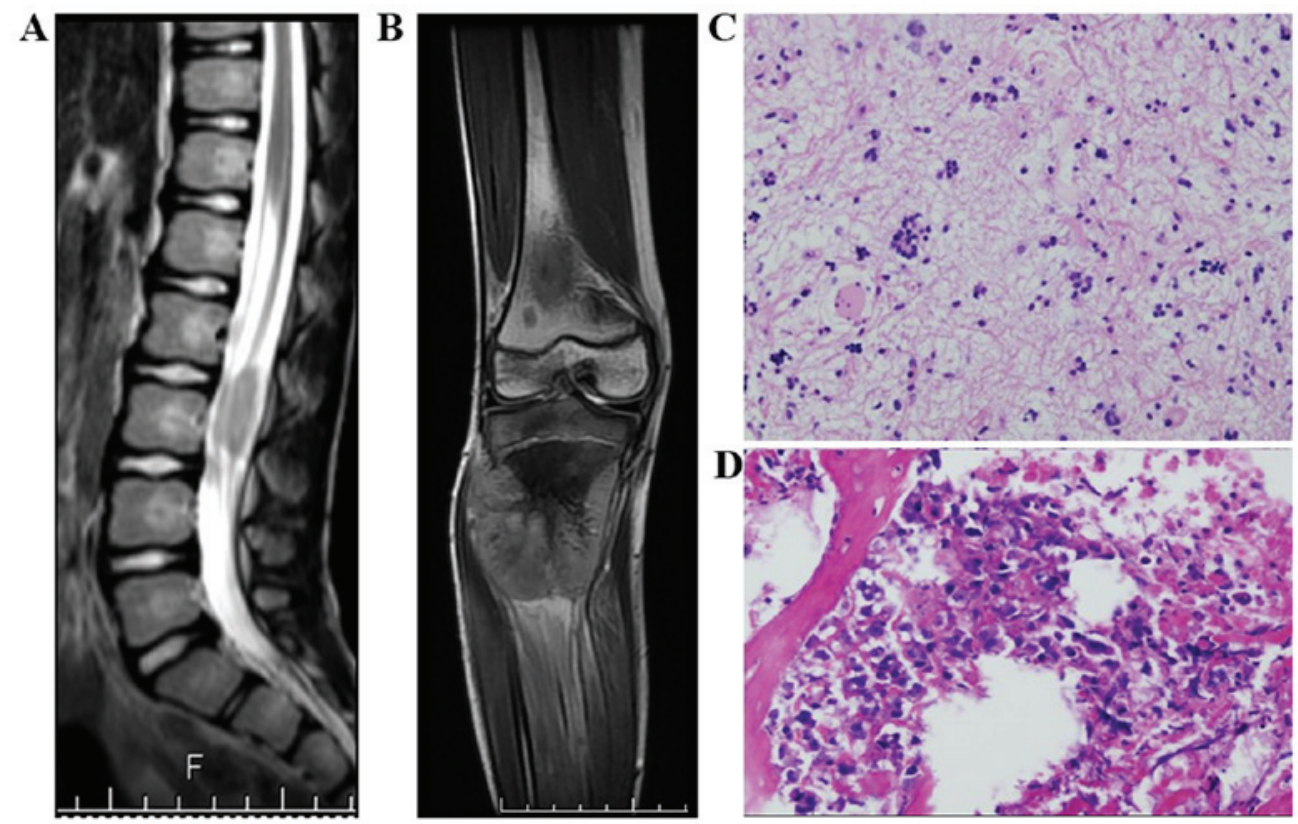

Figure 3. Case 3. (A) Sagittal T2-weighted magnetic resonance imaging (MRI) showing a heterogeneous, isointense mass. (B) Coronal T2-weighted MRI showing a heterogeneous, hyperintense expansible mass. (C) Primary cancer: Astrocytoma at the intradural region of the L3 level (hematoxylin and eosin staining; magnification, x200). (D) Pathological examination confirmed osteoblastic osteosarcoma (hematoxylin and eosin staining; magnification, x200) (d).

for $>20$ years (12). This represents a 10 -fold increased risk of cancer among cancer survivors, compared with the general population (13). In addition, the risk of secondary bone tumors has been reported to be 133 times higher compared with that of the general population, with an estimated 20 -year cumulative risk of $2.8 \%$ (14).

The prognosis of childhood cancer has markedly improved since the 1970s, mainly due to improvements in multidisciplinary therapy, including neoadjuvant chemotherapy, radiation therapy and surgical techniques. However, with the increase in the number of long-term survivors, the prevalence of latent treatment-related adverse effects, such as secondary malignancy, has increased (15).

Medulloblastoma (case 1) accounts for $\sim 10 \%$ of all childhood brain tumors. These tumors occur exclusively in the posterior fossa and have a propensity for leptomeningeal spread. Treatment includes a combination of surgery and radiation therapy (patients aged $>3$ years). Adjuvant 
chemotherapy is recommended for all patients. Patients aged $>3$ years are stratified based on the volume of postoperative metastases (reference value: $1.5 \mathrm{~cm}^{2}$ ) or absence of metastases into standard- and high-risk categories, with long-term survival rates of $\sim 85$ and $70 \%$, respectively. Outcomes are inferior in infants and children aged $<3$ years (16). The patient in case 1 was 4 years old and the primary tumor was resected completely, but intrathecal metastasis was present. Thus, he was deemed a high-risk patient. The radiation dose was reduced to $18 \mathrm{~Gy}$, and an alkylator-based, dose-intensive chemotherapy regimen was administered according to the Children's Oncology Group study in young children (aged 3-7 years) $(16,17)$.

Mediastinal germ cell tumors (case 2) are a rare group of germ cell tumors that account for $<5 \%$ of all germ cell malignancies. Histologically, they may be divided into two categories, namely seminomas and non-seminomas (18). The long-term cure rate of patients with histologically pure seminomas in the mediastinum is almost $90 \%$; only $45 \%$ of patients with mediastinal non-seminoma survive for 5 years, and their outcomes are worse compared with those of patients with seminomas in the gonads or retroperitoneal primary lesions (19). The pathological diagnosis of the primary cancer of the patient in case 2 was non-seminoma. The treatment included conventional chemotherapy with the bleomycin + etoposide + cisplatin and paclitaxel + ifosfamide + cisplatin protocols, with modifications.

Overall, the age-adjusted incidence of anaplastic astrocytoma is 3.5 per million persons/year. The incidence of anaplastic astrocytoma (case 3 ) increases with age, and in children aged $0-15$ years, the rate is 0.9 per million persons/year. The corresponding rates are 2.6 in young adults (16-39 years) and 4.7 in older adults (40-64 years). The overall age-standardized 5- and 10-year relative survival rates of populations with anaplastic astrocytoma are 23.6 and $15.1 \%$, respectively, while the respective 5- and 10-year survival rates in children are 32.1 and $24.6 \%$. The effect of age on survival is present for only the first 3 years post-diagnosis, after which time age no longer affects cancer-specific survival rates (20). The patient in case 3 received localized irradiation and a high-dose intensive chemotherapy protocol that included cyclophosphamide, vincristine, etoposide and cisplatin, put forth by the Pediatric Oncology Group for the treatment of children aged $<3$ years with malignant astrocytoma, with modifications (21).

At least two factors, namely genetic factors and acquired conditions related to treatment modalities, must be considered as the possible causes of secondary malignancy.

Genetic factors include the presence of Li-Fraumeni syndrome (LFS) and retinoblastoma $(22,23)$. Previous studies have demonstrated that childhood cancer survivors with a family history of cancer, particularly the presence of LFS, carry an increased risk of developing secondary cancer $(24,25)$. In all three patients in the present report, the familial history did not fit the clinical diagnostic criteria of LFS or retinoblastoma $(22,23)$.

As regards acquired factors, radiation and chemotherapy have been implicated in the past. Therapy-related solid secondary malignancy is strongly associated with radiation. The risk of solid secondary malignancy is highest when the exposure to radiation occurs at a younger age, and it increases with the total dose of radiation $(13,14)$. Some well-established radiation-related solid secondary malignancies include breast, lung and thyroid cancer, brain tumors, soft tissue sarcomas, and malignant bone tumors, such as Ewing's sarcoma (26). Our patients had not received previous radiation therapy to the anatomical site of the secondary malignancy.

It has been suggested that chemotherapy may cause secondary malignancy in young cancer survivors $(26,27)$. Bhatia et al (26) concluded that the use of alkylating agents and topoisomerase II inhibitors for childhood cancer increases the subsequent risk of secondary tumors. Hawkins et al (28) reported that the risk of alkylating agent-related secondary malignancy is dose-dependent.

Mutagenicity is associated with the ability of alkylating agents to form crosslinks and/or transfer alkyl groups to form DNA monoadducts (29). Topoisomerase II catalyzes the relaxation of supercoiled DNA by covalently binding and transiently cleaving and re-ligating both strands of the DNA helix. DNA topoisomerase II inhibitors stabilize the enzyme-DNA covalent intermediate, decrease the relegation rate, and cause chromosomal breakage (30). In our three cases, alkylating agents (ifosfamide and cyclophosphamide) and a topoisomerase II inhibitor (etoposide) had been administered for the primary cancer. While it is impossible to definitively establish a link between previous chemotherapy and secondary malignancies, an association may well exist.

The interval from the diagnosis of primary malignancy to the development of secondary malignancy may be 8-10 years (27). In our patients, a period of $\sim 7$ years elapsed between the initial diagnosis of primary cancer and the detection of a possible secondary malignancy. Thus, long-term follow-up may be necessary to identify the development of a secondary malignancy in young survivors of malignant tumors in order to monitor latent treatment-related adverse effects.

In conclusion, we herein presented three cases of osteosarcoma developing several years after the primary malignancies. Medical professionals, including orthopedic oncologists, as well as patients, should be aware of the possibility of the occurrence of a secondary malignancy as a potential adverse latent effect. Long-term follow-up is crucial for young survivors, even after successful treatment of the primary cancer.

\section{Acknowledgements}

Not applicable.

\section{Funding}

No funding was received.

\section{Availability of data and materials}

Not applicable.

\section{Authors' contributions}

Conception or design of the work: AS; data collection: AS, NO, TI, NT, JH, CN; data analysis and interpretation: AS, CN; 
drafting the article: AS; critical revision of the article: MA, $\mathrm{MH}$; final approval of the version to be published: HN.

\section{Ethics approval and consent to participate}

Study approval was obtained from the institution, and all investigations were conducted in accordance with the ethical principles of research.

\section{Patient consent for publication}

The patients and their families provided consent for the publication of patient data.

\section{Competing interests}

The authors declare that they have no competing interests.

\section{References}

1. Meyers PA, Schwartz CL, Krailo M, Kleinerman ES Betcher D, Bernstein ML, Conrad E, Ferguson W, Gebhardt M, Goorin AM, et al: Osteosarcoma: A randomized, prospective trial of the addition of ifosfamide and/or muramyl tripeptide to cisplatin, doxorubicin and high-dose methotrexate. J Clin Oncol 23: 2004-2011, 2005.

2. Raymond AK, Ayala AG and Nuutila S: Conventional osteosarcoma. In: World Health Organization classification of tumors: Pathology and genetics; tumors of soft tissue and bone. Fletcher CDM, Unni KK and Mertens F (eds). International Agency for Research on Cancer, Lyon, pp24-70, 2002.

3. Uni KK and Inwards CY: Osteosarcoma. In: Dahlin's bone tumors. 6th edition. Uni KK and Inwards CY (eds). Wolters Kluwer, Lippincott Williams and Wilkins, Philadelphia, pp122-pp157, 2010.

4. Arndt CA and Crist WM: Common musculoskeletal tumors of childhood and adolescence. N Engl J Med 341: 342-352, 1999.

5. Henderson TO, Whitton J, Stovall M, Mertens AC, Mitby P, Friedman D, Strong LC, Hammond S, Neglia JP, Meadows AT, et al: Secondary sarcomas in childhood cancer survivors: A report from the childhood cancer survivor study. J Natl Cancer Inst 99: 300-308, 2007.

6. Smith MA, Seibel NL, Altekruse SF, Ries LA, Melbert DL, O'Leary M, Smith FO and Reaman GH: Outcomes for children and adolescents with cancer: Challenges for the twenty-first century. J Clin Oncol 28: 2625-2634, 2010.

7. Cho Y, Jung GH, Chung SH, Kim JY, Choi Y and Kim JD: Long-term survivals of stage IIb osteosarcoma: A 20-year experience in a single institution. Clin Orthop Surg 3: 48-54, 2011.

8. Bielack SS, Kempf-Bielack B, Heise U, Schwenzer D and Winkler K: Combined modality treatment for osteosarcoma occurring as a second malignant disease. Cooperative German-Austrian-Swiss Osteosarcoma Study Group. J Clin Oncol 17: 1164, 1999.

9. Bielack S, Beck J, Delling G, Gerein V, Grümayer R, Hiddemann W, Jobke A, Jürgens H, Kornhuber G, Kotz R, et al: Neoadjuvant chemotherapy of osteosarcoma. Results of the cooperative studies COSS-80 and COSS-82 after 7 and 5 years. Klin Padiatr 201: 275-284, 1989 (In German).

10. Yonemoto T, Hosono A, Iwata S, Kamoda H, Hagiwara Y, Fujiwara T, Kawai A and Ishii T: The prognosis of osteosarcoma occurring as second malignancy of childhood cancers may be favorable: Experience of two cancer centers in Japan. Int J Clin Oncol 20: 613-616, 2015.

11. Pizzo PA and Poplack DG (eds): Principles and practice of pediatric oncology. Wolters Kluwer, Sixth edition, 2015.
12. Meadows AT, Friedman DL, Neglia JP, Mertens AC, Donaldson SS, Stovall M, Hammond S, Yasui Y and Inskip PD: Second neoplasm in survivors of childhood cancer: Findings from the childhood cancer survivor study cohort. J Clin Oncol 27: 2356-2362, 2009.

13. Neglia JP, Friedman DL, Yasui Y, Mertens AC, Hammond S, Stovall M, Donaldson SS, Meadows AT and Robison LL: Second malignant neoplasms in five-year survivors of childhood cancer: Childhood cancer survivor study. J Natl Cancer Inst 93: 618-629, 2001.

14. Tucker MA, D'Angio GJ, Boice JD Jr, Strong LC, Li FP, Stovall M, Stone BJ, Green DM, Lombardi F, Newton W, et al: Bone sarcomas linked to radiotherapy and chemotherapy in children. N Engl J Med 317: 588-593, 1987.

15. Hudson MM: Late complications after leukemia therapy. Cambridge University Press, pp701-713, 2012.

16. Millard NE and De Braganca KC: Medulloblastoma. J Child Neurol 31: 1341-1353, 2016.

17. Gajjar A, Chintagumpala M, Ashley D, Kellie S, Kun LE, Merchant TE, Woo S, Wheeler G, Ahern V, Krasin MJ, et al: Risk-adapted craniospinal radiotherapy followed by high-dose chemotherapy and stem-cell rescue in children with newly diagnosed medulloblastoma (St Jude Medulloblastoma-96): Long-term results from a prospective, multicentral trial. Lancet Oncol 7: 813-820, 2006.

18. Chetaille B, Massard G and Falcoz PE: Mediastinal germ cell tumors. Anatomopathology, classification, teratomas and malignant tumors. Rev Pneumol Clin 66: 63-70, 2010 (In French).

19. Bokemeyer C, Nichols CR, Droz JP, Schmoll HJ, Horwich A, Gerl A, Fossa SD, Beyer J, Pont J, Kanz L, et al: Extragonadal germ cell tumors of the mediastinum and retroperitoneum: Results from an international analysis. J Clin Oncol 20: 1864-1873, 2002.

20. Smoll NR and Hamilton B: Incidence and relative survival of anaplastic astrocytomas. Neuro Oncol 16: 1400-1407, 2014.

21. Duffner PK, Horowitz ME, Krischer JP, Friedman HS, Burger PC, Cohen ME, Sanford RA, Mulhern RK, James HE, Freeman CR et al: Postoperative chemotherapy and delayed radiation in children less than three years of age with malignant brain tumors. N Engl J Med 328: 1725-1731, 1993.

22. Li FP, Fraumeni JF Jr, Mulvihill JJ, Blattner WA, Dreyfus MG, Tucker MA and Miller RW: A cancer family syndrome in twenty-four kindreds. Cancer Res 48: 5358-5362, 1988.

23. Kay RM, Eckardt JJ and Mirra JM: Osteosarcoma and Ewing's sarcoma in a retinoblastoma patient. Clin Orthop Relat Res 323: 284-287, 1996.

24. Andersson A, Enblad G, Tavelin B, Björkholm M, Linderoth J, Lagerlöf I, Merup M, Sender M and Malmer B: Family history of cancer as a risk factor for second malignancies after Hodgkin's lymphoma. Br J Cancer 98: 1001-1005, 2008.

25. Hisada M, Garber JE, Fung CY, Fraumeni JF Jr and Li FP: Multiple primary cancers in families with Li-Fraumeni syndrome. J Natl Cancer Inst 90: 606-611, 1998.

26. Bhatia S and Sklar C: Second cancers in survivors of childhood cancer. Nat Rev Cancer 2: 124-132, 2002.

27. Longhi A, Ferrari S, Tamburini A, Luksch R, Fagioli F, Bacci G and Ferrari C: Late effects of chemotherapy and radiotherapy in osteosarcoma and Ewing sarcoma patients: The Italian Sarcoma Group Experience (1983-2006). Cancer 118: 5050-5059, 2012.

28. Hawkins MM, Wilson LM, Burton HS, Potok MH, Winter DL, Marsden HB and Stovall MA: Radiotherapy, alkylating agents, and risk of bone cancer after childhood cancer. J Natl Cancer Inst 88: 270-278, 1996.

29. Thirman MJ and Larson RA: Therapy-related myeloid leukemia. Hematol Oncol Clin North Am 10: 293-320, 1996.

30. Corbett AH and Osheroff $\mathrm{N}$ : When good enzymes go bad: Conversion of topoisomerase II to a cellular toxin by antineoplastic drugs. Chem Res Toxicol 6: 585-597, 1993. 